\title{
Obesity-induced inflammatory changes in adipose tissue
}

\author{
Kathryn E. Wellen and Gökhan S. Hotamisligil \\ Department of Genetics and Complex Diseases, Harvard School of Public Health, \\ Boston, Massachusetts, USA
}

\begin{abstract}
Obesity is associated with a state of chronic, low-grade inflammation. Two manuscripts in this issue of the JCI (see the related articles beginning on pages 1796 and 1821) now report that obese adipose tissue is characterized by macrophage infiltration and that these macrophages are an important source of inflammation in this tissue. These studies prompt consideration of new models to include a major role for macrophages in the molecular changes that occur in adipose tissue in obesity.
\end{abstract}

J. Clin. Invest. 112:1785-1788 (2003). doi:10.1172/JCI200320514.

\section{Obesity, diabetes, and inflammation}

Obesity and the associated metabolic pathologies are the most common and detrimental metabolic diseases, affecting over $50 \%$ of the adult population. These conditions are associated with a chronic inflammatory response characterized by abnormal cytokine production, increased acute-phase reactants, and activation of inflammatory signaling pathways (1). This association is not an inconsequential one, at least in experimental models, and is causally linked to either obesity itself or closely linked diseases such as insulin resistance, type 2 diabetes, and cardiovascular disease.

A very interesting feature of the inflammatory response that emerges in the presence of obesity is that it appears to be triggered, and to reside predominantly, in adipose tissue, although other metabolically critical sites may also be involved during the course of the disease (2). The temporal and spatial properties of the inflammatory response in the context of obesity and its complications, the target cell(s) that is critical in

Address correspondence to: Gökhan S. Hotamisligil, Department of Genetics and Complex Diseases, Harvard School Of Public Health, 665 Huntington Avenue, Boston, Massachusetts 02115, USA.

Phone: (617) 432-1950; Fax: (617) 432-1941;

E-mail: ghotamis@hsph.harvard.edu.

Conflict of interest: The authors have

declared that no conflict of interest exists. metabolic dysregulation, and the underlying molecular mechanisms remain as unanswered but critical questions.

\section{Immune features of adipocytes}

Many of us in the research field have examined adipose tissue and adipocytes in a search for the mechanisms underlying obesity and associated diseases. This disease cluster, including type 2 diabetes and atherosclerosis (among others), features abnormalities of both metabolic and inflammatory pathways. While the role of adipocytes in metabolic pathways is clear, little is understood about their role in inflammation. Following careful examination, it has been shown that adipocytes and diverse types of immune cells such as $\mathrm{T}$ cells and macrophages possess similar roles in pathways such as complement activation and inflammatory cytokine production $(3,4)$. Here, the focus will be on the striking overlap between the biology of adipocytes and that of macrophages.

Adipocyte precursors have potent phagocytic capacity and can be transformed into macrophage-like cells in response to appropriate stimuli (5). Many genes that are critical to adipocytes, including those encoding transcription factors, cytokines, inflammatory molecules, fatty acid transporters, and scavenger receptors, are also expressed in macrophages and have an important role in macrophage biology $(6,7)$. In fact, at least in our view, it is challenging to find exceptions to this functional and molecular overlap between fat cells and macrophages.

\section{Macrophages in obesity}

Earlier transcriptional profiling experiments performed in adipose tissue from lean and obese animal models (8), or following treatment of obese animals with members of the thiazolidinedione class of antidiabetic agents (9), have pointed to striking regulation of a large repertoire of inflammatory genes in adipose tissue. Two articles in this issue of the JCI illustrate that macrophage infiltration into adipose tissue in obesity could be integral to these inflammatory changes $(10,11)$. Both of these studies have principally emerged from large-scale gene-expression analysis. $\mathrm{Xu}$ and colleagues directly compared gene expression in multiple tissues between five obese mouse models and their lean controls (10). Weisberg et al. chose to profile gene expression in the white adipose tissue of mice of varying degrees of obesity, in order to identify correlations between gene expression and the degree of obesity (11). In support of earlier studies, both approaches indicate that the largest class of genes significantly regulated in obesity consists of macrophage and inflammatory genes in white adipose tissue.

In these articles, both groups considered the possibility that the cellular source of these inflammatory changes may not be only adipocytes, but also reticuloendothelial cells present in adipose tissue or perhaps adipocyte precursors $(10,11)$. The studies to address this possibility led to the observations that some inflammatory responses took place outside of adipocytes in obesity, and that significantly higher numbers of macrophages infiltrated into the expanding adipose tissue. Coming to this conclusion, however, was not easy, precisely due to the similarities of macrophages to adipocytes and pre- 


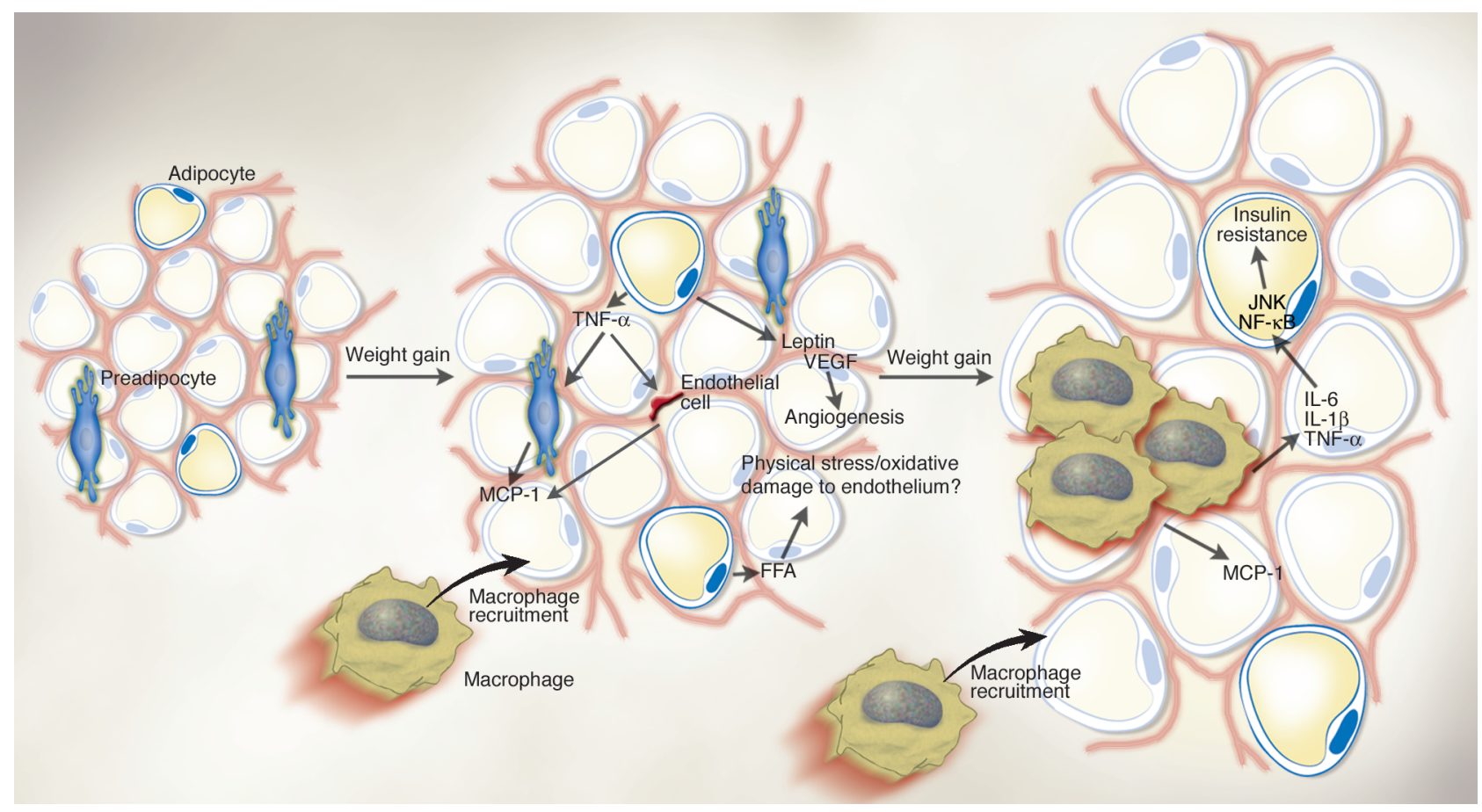

Figure 1

Obese adipose tissue is characterized by inflammation (1) and progressive infiltration by macrophages as obesity develops (10, 11). Changes in adipocyte and fat pad size lead to physical changes in the surrounding area and modifications of the paracrine function of the adipocyte. For example, in obesity, adipocytes begin to secrete low levels of TNF- $\alpha$, which can stimulate preadipocytes to produce monocyte chemoattractant protein-1 (MCP-1) (10). Similarly, endothelial cells also secrete MCP-1 in response to cytokines. Thus, either preadipocytes or endothelial cells could be responsible for attracting macrophages to adipose tissue. The early timing of MCP-1 expression prior to that of other macrophage markers during the development of obesity also supports the idea that it is produced initially by cells other than macrophages (10). Increased secretion of leptin (and/or decreased production of adiponectin) by adipocytes may also contribute to macrophage accumulation by stimulating transport of macrophages to adipose tissue (17) and promoting adhesion of macrophages to endothelial cells, respectively (15). It is conceivable, also, that physical damage to the endothelium, caused either by sheer size changes and crowding or oxidative damage resulting from an increasingly lipolytic environment, could also play a role in macrophage recruitment, similar to that seen in atherosclerosis. Whatever the initial stimulus to recruit macrophages into adipose tissue is, once these cells are present and active, they, along with adipocytes and other cell types, could perpetuate a vicious cycle of macrophage recruitment, production of inflammatory cytokines, and impairment of adipocyte function.

adipocytes, as mentioned above. To resolve the critical question of accurate identification of the macrophages, the investigators performed a series of elegant and rigorous experiments. Xu et al. began by separating white adipose tissue into a buoyant fraction containing adipocytes and a pelleted stromal-vascular fraction consisting of nonadipocyte cells in the adipose tissue, such as preadipocytes and macrophages. They found that several inflammatory genes chosen from their microarray analysis were in fact expressed predominantly in the stromal-vascular fraction (10). The challenge was then to distinguish between adipocyte precursors and macrophages in this fraction. Histological examination revealed that clusters of small, nucleated cells were present in obese, but not in lean, adipose tissue, and that these clusters became larger and more numerous as the animals aged and gained weight. Staining for F4/80, a macrophagespecific antigen, was also prominent in the obese fat pads as compared with those of lean mice. Experiments in cultured clonal preadipocytes confirmed that the same inflammatory genes identified in the stromal-vascular fraction of white adipose tissue were not expressed in these cells. However, upon stimulation with TNF- $\alpha$, an inflammatory cytokine that is overexpressed in obesity and contributes to insulin resistance, preadipocytes did express some inflammatory genes, suggesting that they too might mount a similar inflammatory response under select conditions.
Weisberg and colleagues also stained for the F4/80 antigen and found that the percentage of $\mathrm{F} 4 / 80^{+}$cells correlated positively with average adipocyte size (11). To confirm that the $\mathrm{F} 4 / 80^{+}$ cells were in fact macrophages, the authors first used FACS to separate $\mathrm{F} 4 / 80^{+}$cells from $\mathrm{F} 4 / 80^{-}$cells in the stromal-vascular fraction of $o b / o b$ mouse fat pads. The F $4 / 80^{+}$cells were found to express several other macrophage-specific markers. Taking advantage of the fact that macrophages originate in the bone marrow, the authors subsequently lethally irradiated mice expressing the CD45.2 leukocyte marker and transplanted bone marrow from CD45.1-expressing mice. After placing the mice on a high-fat diet, the authors observed that $85 \%$ of the $\mathrm{F} 4 / 80^{+}$cells in the adipose tissue were 
in fact carrying the donor marker and, thus, were bone marrow derived. Furthermore, in Csf1 ${ }^{o p / o p}$ mice, which lack macrophages, the presence of $\mathrm{F} 4 / 80^{+}$cells was greatly reduced as compared with that in wild-type mice. However, these animals were not studied under obese conditions. Importantly, Weisberg et al. also provide evidence that macrophage infiltration of adipose tissue is characteristic of human obesity, by determining that both BMI and average adipocyte size were significant predictors of macrophage accumulation in adipose tissue, as assessed by the percentage of $\mathrm{CD}^{6} 8^{+}$cells.

\section{Inflammation and mechanisms of type 2 diabetes: emerging questions} Studies in the past decade left little doubt that inflammatory pathways are critical in the mechanisms underlying insulin resistance and type 2 diabetes, at least in experimental models $(2,12-14)$. Evidence has started to mount that this is also the case in humans. Critical questions include the mechanisms by which the inflammatory response is triggered and maintained in obesity, and how this results in deteriorated metabolic homeostasis in general and glucose metabolism in particular. Regarding the latter question, a good deal of progress has been made in molecular characterization of the cross-talk mechanisms between insulin receptor signaling and inflammatory pathways, providing insight into the development of insulin resistance and type 2 diabetes $(2,12)$. However, the signals and mechanisms that trigger the inflammatory response are not well understood. The results of Xu et al. (10) and Weisberg et al. (11) stimulate consideration of new models that take into account macrophage infiltration into adipose tissue and the interactions between adipocytes and macrophages (Figure 1).

What attracts macrophages to the adipose tissue, as opposed to other sites, in obesity? Does the initial inflammatory response emerge from the adipocyte and further propagate with the recruitment of macrophages, or do macrophages first infiltrate the adipose tissue and initiate the inflammatory response themselves? Perhaps the most likely possibility is that the inflammatory reaction and its consequences are the cumulative results of the interactions between adipocytes and macrophages in the context, and under the architectural constraints, of adipose tissue (Figure 1). Might other cell types, such as preadipocytes or endothelial cells, be involved? Could the significant changes in the turnover rate of other bioactive and hazardous cargo released by the fat cells expose the vast vasculature in adipose tissue to an environment of oxidative or other injury to which the macrophages respond? This scenario might be synonymous with the endothelial injury and macrophage recruitment that occur during atherosclerosis, except that in this case it could disturb insulin action.

Another key question is what proportion of these inflammatory genes are causally linked to particular metabolic outcomes. In this regard, it might be instrumental to dissect the gene clusters involved in models where manipulation of inflammatory pathways is known to be connected to a clear metabolic outcome. For example, exploring the contributions of TNF- $\alpha$, adiponectin, JNK, and inhibitor of NF- $\mathrm{KB}$ kinase $\beta$ (IKK $\beta$ ) $(2,12,13,15)$ to adipose tissue inflammation in mice with null mutations in these genes might provide insights into the aspects of the inflammatory response that are relevant to insulin action. These or similar studies could also be directed to address the potential involvement of inflammation in other pathologies associated with obesity. While these and many other questions are being answered, it is likely that a rich array of mechanistic and therapeutic developments will emerge from studies of the inflammatory pathways active in obesity and associated disorders.

\section{Evolutionary perspectives}

One might consider a few evolutionary prospects regarding the intimate relationship between the immune and metabolic responses. First, the functional structures that control key metabolic and immune functions have evolved from common ancestors. The best example of this is the Drosophila fat body, which contains the mammalian homologs of liver, the hemapoietic system, and other immune components. Recently, it was shown that this site also corresponds to mammalian adipose tissue (16). As these specialized cells differentiate into distinct functional units or organs, they carry with them their developmental heritage. Hence, it is possible to envision a scenario where common pathways regulate both metabolic and immune functions through the utilization of common key regulatory molecules.

Second, such a closely linked configuration and coordinated regulation of metabolic and immune responses is likely to be advantageous, since the organism needs to organize and redistribute its metabolic resources during the mounting of an immune or inflammatory response. In fact, the most primitive responses integrate the pathogenand nutrient-sensing pathways such that nutrients can evoke immune responses and pathogens can regulate metabolic responses. Unfortunately, caught in this crossfire is the modern human. The very metabolic adaptations with which we have managed to survive as a species now threated to defeat us as we respond to the stresses and indulge in the products of cultural and industrial globalization.

1. Hotamisligil, G.S. 2003. Inflammation, TNFo and insulin resistance. In Diabetes mellitus. D. LeRoith, S.I. Taylor, and J.M. Olefsky, editors Lippincott-Raven Publishers. Philadelphia, Pennsylvania, USA. In press.

2. Hirosumi, J., et al. 2002. A central role for JNK in obesity and insulin resistance. Nature. 420:333-336.

3. Hotamisligil, G.S., Shargill, N.S., and Spiegelman, B.M. 1993. Adipose tissue expression of tumor necrosis factor- $\alpha$ : direct role in obesity-linked insulin resistance. Science. 259:87-90.

4. Rosen, B.S., et al. 1989. Adipsin and complement factor D activity: an immune-related defect in obesity. Science. 244:1483-1487.

5. Charriere, G., et al. 2003. Preadipocyte conversion to macrophage. Evidence of plasticity. J. Biol. Chem. 278:9850-9855.

6. Tontonoz, P., Nagy, L., Alvarez, J.G., Thomazy, V.A., and Evans, R.M. 1998. PPARgamma promotes monocyte/macrophage differentiation and uptake of oxidized LDL. Cell. 93:241-252.

7. Makowski, L., et al. 2001. Lack of macrophage fatty-acid-binding protein aP2 protects mice deficient in apolipoprotein E against atherosclerosis. Nat. Med. 7:699-705 
8. Soukas, A., Cohen, P., Socci, N.D., and Friedman, J.M. 2000. Leptin-specific patterns of gene expression in white adipose tissue. Genes Dev. 14:963-980

9. Way, J.M., et al. 2001. Comprehensive messenger ribonucleic acid profiling reveals that peroxisome proliferator-activated receptor gamma activation has coordinate effects on gene expression in multiple insulin-sensitive tissues. Endocrinology. 142:1269-1277.

10. Xu, H., et al. 2003. Chronic inflammation in fat plays a crucial role in the development of obesity-related insulin resistance. J. Clin. Invest.
112:1821-1830. doi:10.1172/JCI200319451.

11. Weisberg, S.P., et al. 2003. Obesity is associated with macrophage accumulation in adipose tissue. J. Clin. Invest. 112:1796-1808. doi:10.1172/ JCI200319246.

12. Yuan, M., et al. 2001. Reversal of obesity- and diet-induced insulin resistance with salicylates or targeted disruption of Ikkbeta. Science. 293:1673-1677.

13. Uysal, K.T., Wiesbrock, S.M., Marino, M.W., and Hotamisligil, G.S. 1997. Protection from obesityinduced insulin resistance in mice lacking TNFalpha function. Nature. 389:610-614.
14. Perreault, M., and Marette, A. 2001. Targeted dis ruption of inducible nitric oxide synthase protects against obesity-linked insulin resistance in muscle. Nat. Med. 7:1138-1143.

15. Maeda, N., et al. 2002. Diet-induced insulin resistance in mice lacking adiponectin/ACRP30. Nat. Med. 8:731-737

16. Tong, Q., et al. 2000. Function of GATA transcription factors in preadipocyte-adipocyte transition. Science. 290:134-138.

17. Sierra-Honigmann, M.R., et al. 1998. Biological action of leptin as an angiogenic factor. Science 281:1683-1686.

\section{A radical explanation for glucose-induced $\beta$ cell dysfunction}

\section{Michael Brownlee}

Diabetes Research Center, Albert Einstein College of Medicine, New York, New York, USA

The development of type 2 diabetes requires impaired $\beta$ cell function. Hyperglycemia itself causes further decreases in glucose-stimulated insulin secretion. A new study demonstrates that hyperglycemia-induced mitochondrial superoxide production activates uncoupling protein 2 , which decreases the ATP/ADP ratio and thus reduces the insulin-secretory response (see the related article beginning on page 1831). These data suggest that pharmacologic inhibition of mitochondrial superoxide overproduction in $\beta$ cells exposed to hyperglycemia could prevent a positive feed-forward loop of glucotoxicity that drives impaired glucose tolerance toward frank type 2 diabetes.

J. Clin. Invest. 112:1788-1790 (2003). doi:10.1172/JCI200320501.

\section{The diabetes epidemic and its consequences}

Diabetes and impaired glucose tolerance currently affect an estimated 29 million people in the US (1). For those born in 2000, the estimated lifetime risk of developing diabetes is 36\% (2). People with diabetes have large reductions in life expectancy and in quality of life (2), due to diabetes-specific microvascular complications in the retina, renal glomerulus, and peripheral nerve, and to extensive atherothrombotic macrovascular disease affecting arteries that supply the heart, brain, and lower extremities. It has

Address correspondence to: Michael Brownlee, Diabetes Research Center, Albert Einstein College of Medicine, 1300 Morris Park Avenue, New York, New York 10461, USA. Phone: (718) 430-3636;

Fax: (718) 430-8570;

E-mail: Brownlee@aecom.yu.edu.

Conflict of interest: The author has declared that no conflict of interest exists.

Nonstandard abbreviations used: tricarboxylic acid (TCA); reduced flavin adenine dinucleotide $\left(\mathrm{FADH}_{2}\right)$; uncoupling protein (UCP). been estimated that up to $70 \%$ of patients with acute myocardial infarction have either diabetes or impaired glucose tolerance (3).

As a consequence of its microvascular pathology, diabetes is the leading cause of blindness, end-stage renal disease, and a variety of debilitating neuropathies. Diabetics are the fastest-growing group of renal dialysis and transplant recipients, and in the US, their 5-year survival rate is only 21 percent, worse overall than that for all forms of cancer combined. Over $60 \%$ of diabetic patients suffer from neuropathy, which accounts for $50 \%$ of all nontraumatic amputations in the US (4).

\section{Insulin resistance, $\beta$ cell function, and the natural history of type 2 diabetes}

Both genetic and environmental factors (mainly obesity) contribute to insulin resistance. Recent work with tissue-conditional knockouts of both Glut4 and the insulin receptor in mice have shown that adipose tissue plays a central role in the pathogenesis of insulin resistance, and that there is significant crosstalk among insulin target tissues (5).

Insulin resistance induces a compensatory increase in $\beta$ cell mass, which in many people results in normal glucose levels. In other people, intrinsic defects in this compensatory $\beta$ cell response prevent adequate compensation, and impaired glucose tolerance or type 2 diabetes occurs. Impaired glucose tolerance leads to type 2 diabetes in a significant number of people, and type 2 diabetes, in turn, becomes progressively unresponsive to oral antidiabetic agents, until treatment with insulin is necessary.

\section{Glucose toxicity and the decline of $\beta$ cell function}

Hyperglycemia is widely recognized as the causal link between diabetes and diabetic complications (6). More recently, adverse effects of hyperglycemia on insulin target tissues and on pancreatic $\beta$ cells have also been recognized, and this phenomenon has been termed "glucotoxicity." Chronic hyperglycemia has been shown to induce multiple defects in $\beta$ cells, including early decreases in glucose-stimulated insulin secretion, and late irreversible changes in insulin-gene transcription and $\beta$ cell mass $(7,8)$. In patients with impaired glucose tolerance, lowering of glucose levels dramatically reduces the progression to type 2 diabetes (9), suggesting that glucotoxicity plays a major role in this transition.

The central role of mitochondria in glucose-stimulated insulin secretion Pancreatic $\beta$ cells sense the ambient plasma glucose concentration because (a) the high- $K_{m}$ glucose transporter GLUT2 facilitates rapid equilibration across the cell membrane, and (b) the 